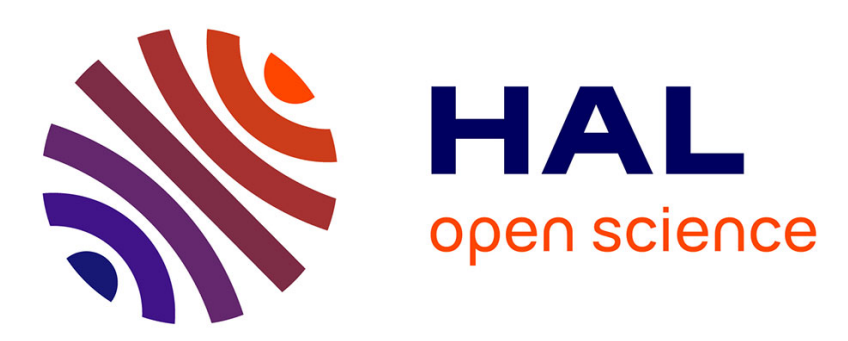

\title{
Neural Mechanisms of Social Emotion Perception: An EEG Hyper-scanning Study
}

\author{
Li Zhu, Fabien Lotte, Gaochao Cui, Junhua Li, Changle Zhou, Andrzej
}

Cichocki

\section{- To cite this version:}

Li Zhu, Fabien Lotte, Gaochao Cui, Junhua Li, Changle Zhou, et al.. Neural Mechanisms of Social Emotion Perception: An EEG Hyper-scanning Study. Cyberworlds 2018, Oct 2018, Singapore, Singapore. hal-01863881

\section{HAL Id: hal-01863881 \\ https://hal.inria.fr/hal-01863881}

Submitted on 29 Aug 2018

HAL is a multi-disciplinary open access archive for the deposit and dissemination of scientific research documents, whether they are published or not. The documents may come from teaching and research institutions in France or abroad, or from public or private research centers.
L'archive ouverte pluridisciplinaire HAL, est destinée au dépôt et à la diffusion de documents scientifiques de niveau recherche, publiés ou non, émanant des établissements d'enseignement et de recherche français ou étrangers, des laboratoires publics ou privés. 


\section{Neural Mechanisms of Social Emotion Perception: An EEG Hyper-scanning Study}

\author{
Li Zhu \\ Cognitive Science Department \\ Xiamen University \\ Xiamen, China \\ zhulibrain@gmail.com \\ Junhua $\mathrm{Li}^{*}$ \\ Singapore Institute for Neurotechnology (SINAPSE) \\ National University of Singapore \\ Singapore, Singapore \\ juhalee.bcmi@gmail.com \\ Andrzej Cichocki \\ Skoikovo Institute of Science and Technology (Skoltech) \\ Moscow, Russia \\ Department of Informatics \\ Nicolaus Copernicus University \\ Torun, Poland \\ School of Computer Science and Techology \\ Hanzhou Dianzi University \\ Hangzhou, China \\ A.Cichocki@skoltech.ru
}

\author{
GaoChao Cui \\ Electronics Engineering Department \\ Saitama Institute of Technology \\ Fukaya, Japan \\ cuigaochao@gmail.com
}

\author{
Changle Zhou * \\ Cognitive Science Department \\ Xiamen University \\ Xiamen, China \\ dozero@xmu.edu.cn
}

\begin{abstract}
EEG-based hyper-scanning refers to two or more subjects engaged in a task together or performing the same action together while neurophysiological signals are simultaneously recorded from them. This is one of the manners for investigating between-subject neural activities involved in social interactions. Emotion perception plays an important role in human social interactions. Interaction and emotional state influence each other. In this study, we aim to investigate how between-subject interaction modulates emotion perception based on event related potentials (ERPs), connectivity analysis and classification analysis. We found that there are distinct differences appearing between paired subjects who performed the task together, which are early ERP components ( $\mathbf{N 2 5 0}$ and N400), late ERP components (P1500 and N1500), and the greater amplitude in N250 for the seconding responding subject compared to the first one. In the exploration of connectivity using phase locking value (PLV), we found that there are significant differences among different frequency bands for each subject under positive and negative stimuli and the significant difference of hyper-connectivity existed in the gamma frequency band between positive and negative stimulus trials. In the classification analysis, we compared the hyper-features for
\end{abstract}

The work described in this paper was supported by the National Nature Science Foundation of China (No.61673322), and the National Key Basic Research Program of China (No.2013CB329502). This research was also partially supported by the Ministry of Education and Science of the Russian Federation (grant 14.756.31.0001) and the Polish National Science Center (grant 2016/20/W/N24/00354. This work was also supported by the Japanese Society for the Promotion of Science (JSPS).).

$*$ indicates the corresponding author two individual subjects separately, the performance was improved when hyper-features of the PLV was employed compared to the features of power spectrum density.

\section{INTRODUCTION}

Human being is the sum of all social relations, said by Karl Marx [1].Our daily lives constitute a social world in which communicating with each other is an everyday challenge [2].A fundamental feature of social life is social interaction, or the ways where people act with other persons and react to how other people are acting [3]. It has been found that the quality and quantity of individual social interaction was relevant not only to mental health but also to morbidity and mortality, Moreover, the impact of social interactions on the risk for mortality is comparable to the well-established risk factors for mortality [4], [5]. Although the social nature of humans has been evidenced for thousands of years, the investigation of brain activity during social interactions was initiated a few decades ago in the field of neuroscience [6].

Hyper-scanning has been utilized to investigate brain-to-brain relationships between persons with signal modalities such as fMRI (functional magnetic resonance imaging), fNIRS (functional near infrared spectroscopy) and EEG (electroencephalography) [7], [8], [9], [10], [11]. TThe first exploratory experiment of Hyper-scanning was implemented by Montague 
A

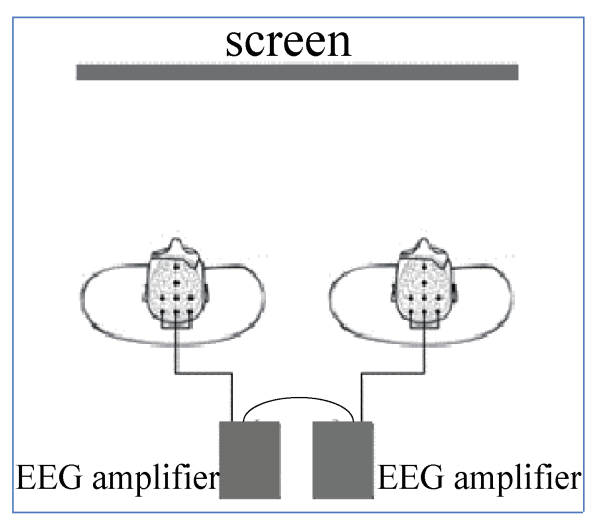

B

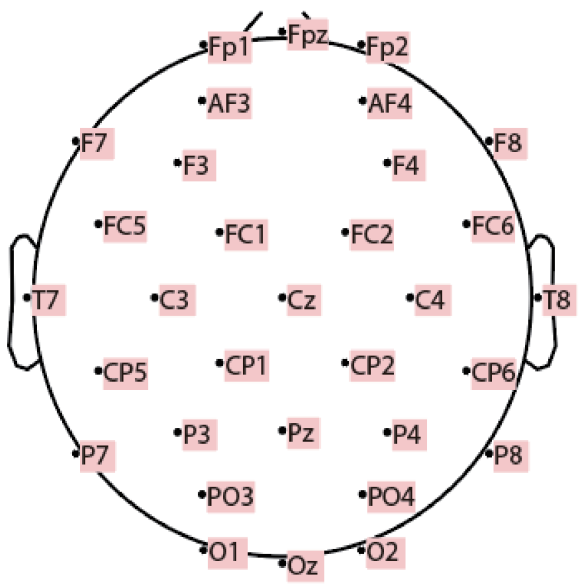

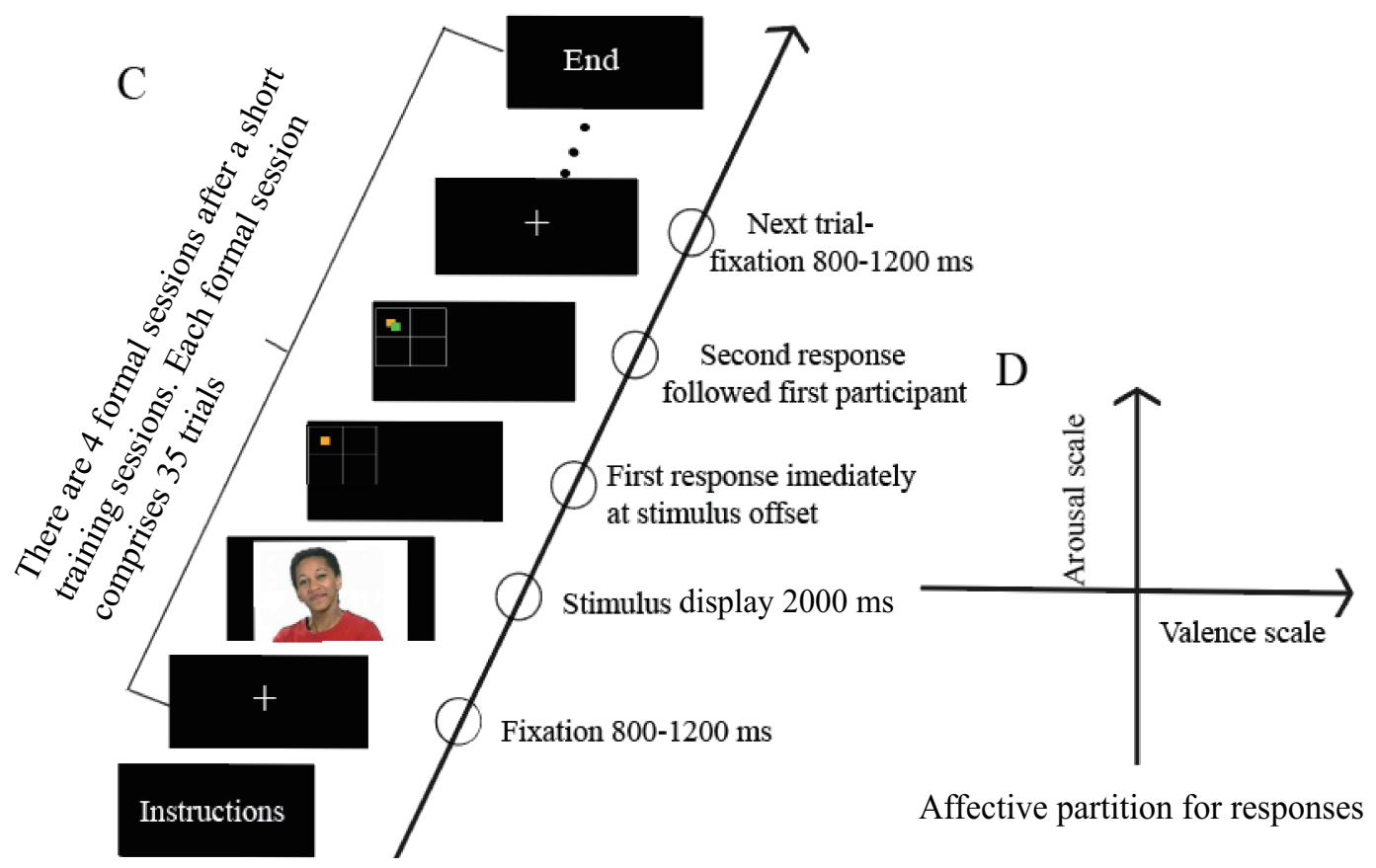

Fig. 1. Experiment settings. (A) shows the experiment setup. (B) gives the layout of channel locations. (C) demonstrates the experiment time-line (D) describes the response panel coordinate.

et al. [12] and the authors presented a game of deception between pairs of subjects through fMRI-based hyper-scanning. $\mathrm{Xu}$ Cui et al. first utilized NIRS device for simultaneous measurements of brain activity of two persons [7] and found that the coherence between signals in the right superior frontal cortex was increased in the case of cooperation. However, this was not observed in the case of competition. Babiloni et al. [13] were the first group to use hyper-scanning with EEG applying the game theory of prisoner's dilemma. They found the most activated region under this task is medial prefrontal cortex. The same group of authors carried on the similar study and reported that the orbital frontal cortex was the most activated region two years later. Based on the pioneering explorations of Hyper-scanning in different specific fields, several experimental paradigms with hyperscanning methodology have been performed in the particular field including motor interactions [14], [15], [16], [17], game theory [8], [18], [19], [20] and economics [21], [22], [23] et al. We focus on EEG-based hyper-scanning technique since EEG experiments are relatively inexpensive, have higher temporal resolution and can be set up in a more naturalistic environment to measure cognitive and motor interactions [24], [25], [26], [27], [28], [29].

Certain emotions-feelings which start with a stimulus and often involve psychological changes and a desire to engage in specific actions come into being during our social interactions. To have a good knowledge of social interaction, it is important to understand how these emotions emerge and how they have influence on and are impacted by social relationship [30], [3]. For instance, [31], [32], [33] have reported emotional synchronization of subjects before and during music production by means of hyper-scanning. [34], [35] proposed the analysis to 
understand the interpersonal influence on the basis of partners facial expressions. The Results suggested that the transmission of affective emotions (with attentional strategies) increased the baseline of social interactions. These results encourage to carry out on human social emotional interactions research with hyper-scanning.

In our study, we have investigated the neural mechanisms of social emotion perception with EEG hyper-scanning. We aim to find out how the brain activity varies on the basis of emotional stimuli between each pair of subjects, with a hypothesis that an enhanced synchronization emotional perception of brains in social interactions. The remaining parts of this paper are organized as follows. Section 2 describes our proposed experiment design and data processing methods. Section 3 presents the performance and comparison results of Event Related Potential (ERP), synchronization with Phase Locking Value (PLV) and the classification methods. Finally, a brief summary is drawn in section 4 .

\section{MATERIALS AND METHODS}

\section{A. Experiment design and data collection}

The goal of our experimental framework was to find out the neural mechanisms of emotion perception between a pair of subjects. In the experiment procedure (shown in Fig.1 (C, D)), two participants simultaneously watched emotional videos and were asked to rate each clip on basis of valence and arousal level of the depicted emotion. Firstly, a short training session was conducted, to familiarize participants with the procedure. Subsequently, four experimental sessions were carried out with self-paced breaks between them. Participants gave their responses by rating their emotion after each stimulus through two connected iPad devices. The ratings were displayed shortly on the screen before starting the next trial. We took 160 short video clips as stimuli from the Cambridge Universitys Mind Reading emotions library which contains actors replaying different emotions through facial expressions. For EEG recordings (show in Fig.1 (A, B)), the signal was acquired through two sets of 32 electrodes (Ag/AgCl, Biosemi) placed accordingly to standard 10-20 montage. Data were gathered with $512 \mathrm{~Hz}$ by two Biosemi amplifiers connected to synchronize the recording. A total of 12 participants (one female) were divided into six pairs to perform the experiment. All subjects gave their written informed consent to participate in the experiment. In the experiment, the participants alternated the order in which they gave responses. For instance, for a pair of participants, subject $\mathrm{A}$ and $\mathrm{B}$, subject $\mathrm{A}$ gives the first ratings on the stimulus in the first two sessions and then subject $\mathrm{B}$ will make the first ratings on the stimulus in the following two sessions.

For EEG signal processing, the data were band pass filtered with the cutoff frequencies of $1 \mathrm{~Hz}$ and $50 \mathrm{~Hz}$. We used a common average reference for the data analysis and applied Independent Component Analysis (ICA) based on EEGLAB [36], [37] to decompose the EEG data into independent components. By visual inspection and the Adjust (i.e., a plugin in EEGLAB), the components corresponding to artifacts such as eye links and movements were rejected and the artifact removal EEG signals were reconstructing based on remaining ICA components. And then, we extracted the EEG signal from the following five frequency bands: delta $(1-3 \mathrm{~Hz})$, theta (4-7 $\mathrm{Hz})$, alpha $(8-13 \mathrm{~Hz})$, beta $(14-30 \mathrm{~Hz})$ and gamma $(31-50 \mathrm{~Hz})$.

\section{B. Estimation of methods}

1) ERP: Epochs, time locked to stimuli, were extracted from $100 \mathrm{~ms}$ prior to the onsets of stimuli to $100 \mathrm{~ms}$ before the ends of stimuli. Those epochs with obvious residual artifacts were excluded for further analysis. The epochs whose amplitudes exceeded $200 \mu \mathrm{V}$ or changes were greater than 100 $\mu \mathrm{V}$ were also discarded.

2) Synchronization: In order to estimate the intra- and inter- brain patterns, we employed the PLV to investigate task-induced changes in synchronization of neural activity from EEG data. The PLV has been shown to have a good performance in hyper-scanning connection analysis, especially when only small samples are available [38], [39]. The original definition of PLV by Lachaux [42] [40] is estimated by below formula

$$
P L V_{n}=\frac{1}{N}\left|\sum_{k=1}^{N} e^{\left.\left.i\left(\phi^{(} t, k\right)-\psi^{(} t, k\right)\right)}\right|
$$

where $\mathrm{N}$ is the number of trials, $\left.\phi^{(} t, n\right)$ and $\left.\psi^{(} t, n\right)$ are the phase values of channel $\phi$ and $\psi$ for the trial $\mathrm{n}$ at the time t. The range of PLV is from 0 to 1 where 1 indicates perfect phase locking and 0 indicates no phase locking. This form PLV is related to the inter-trial variance of the phase difference, $\sigma_{\phi-\psi}^{2}$, followed the relationship $P L V_{n}=1-\sigma_{\phi-\psi}^{2}$. It is only suitable for event-related analysis since this form of $P L V_{n}$ is based on the phase difference across trials.

We used the variant of the equation (1) that has been frequently used in EEG hyper-scanning studies by averaging the instantaneous phase differences over time within one single trial.

$$
\text { Hyper_PLV } V_{n}=\frac{1}{T}\left|\sum_{n=1}^{T} e^{\left.\left.i\left(\phi^{(} t, n\right)-\psi^{(} t, n\right)\right)}\right|
$$

where $\mathrm{T}$ is the number of time points. Formula (2) is a measure of intra-trial consistency of the phase difference between channels. This difference makes the formula (2) has clear interpretation of EEG hyper-scanning.

Specifically, we computed the PLV and hyper_PLV value between the EEG signals for each segment. Therefore, we obtained the $32 \times 32$ PLV matrix denoting the synchronization between channels of the interacting individuals and $1024 \times 64 \times 64 \times 2$ Hyper_PLV (data points $\times$ channel $\times$ channel $\times$ label) the high-dimensional matrix. By averaging all these matrices corresponding to all epochs, we computed the intra- and inter-brain synchrony as a representative of each paired participants' experiment duration.

In order to study the differences in the intra-brain synchrony between different types (i.e., positive and negative) of emotional stimuli, we performed the statistical comparisons across 


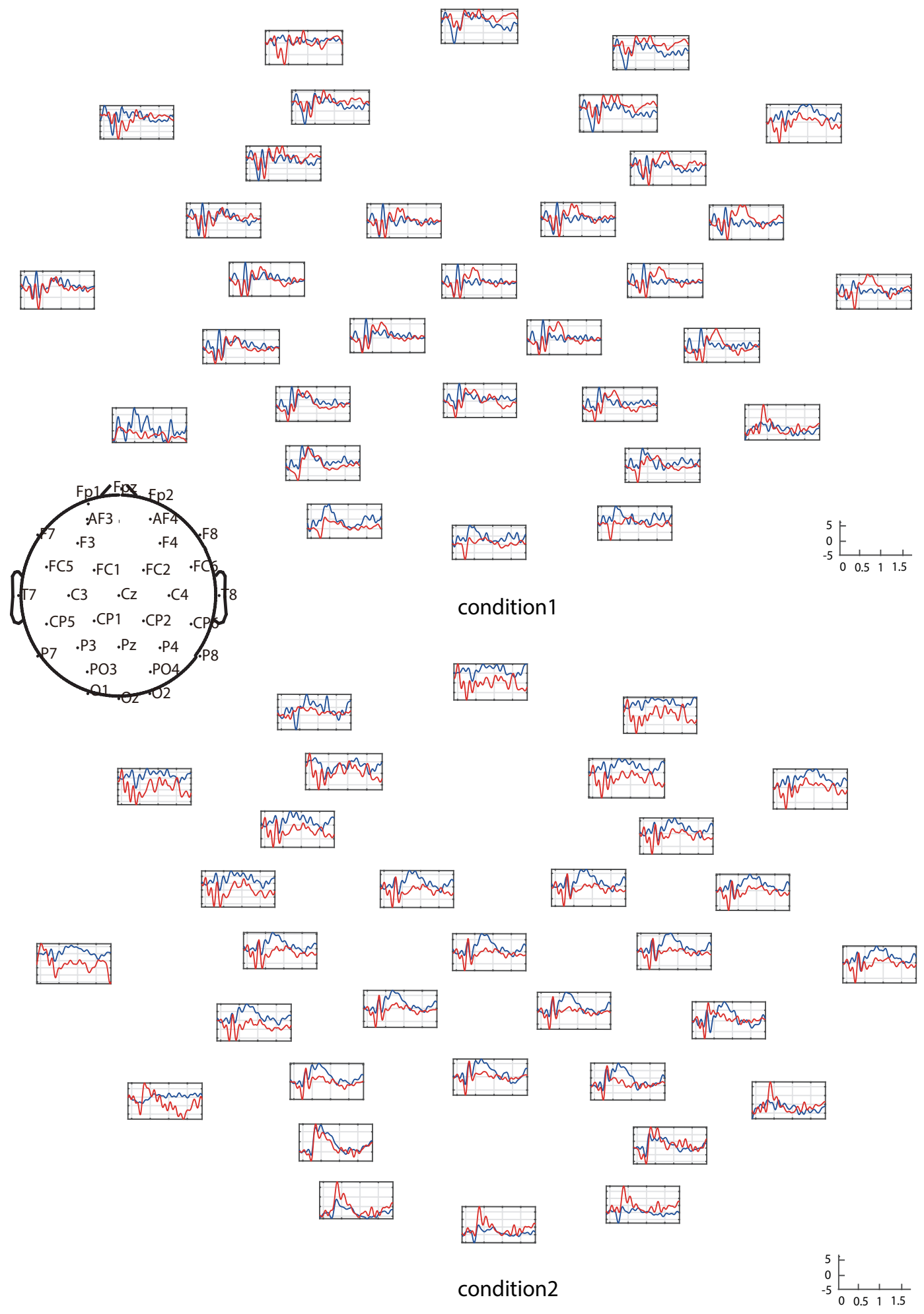

Fig. 2. ERP results. Blue lines indicate the one of the paired participant while red lines represent the other participant. The blue labeled participant makes first response in condition 1 and in condition 2, the response order of the participants changes and the blue lines are labeled for the other participant who makes first response.

each edge vector (representing PLV values between channels) to determine significantly different edges between emotion types (i.e., positive vs. negative). For inter-brain synchrony, we obtained inter-brain synchrony matrices consisting of significantly different edges by the Wilcoxon rank test with a strict threshold of p-value at 0.01 . 
3) Classification: Classification evaluation has been widely used to distinguish the emotions for single-subject EEG experiments in related works. For instance, in [41] they achieved an accuracy of $65 \%$ for both valence and arousal using the wavelet entropy of 3 to 12 seconds signal segments. In [42], they introduced the discriminate graph regularized extreme learning to find the relationship between EEG signals and human emotional states and the average accuracy of the method is $80.25 \%$, while the accuracy of Support Vector Machine (SVM) was $76.62 \%$. From the same lab, they combined eye movements and EEG to enhance emotion recognition and the best accuracy achieved by the proposed fuzzy integral fusion strategy was $87.59 \%$ [43], exceeding the one using eye movements $(77.80 \%$ ) and EEG data (78.51\%). Whereas in hyper-scanning emotion study, researchers focus on the information flow between each subjects [6], [7], [8], [9], [10], [11], [12], [7], [13], [14], [15], [16], [17], [8], [18], [19], [20], [21]. There is no report on the hyper-scanning based emotion classification analyses.

In our study, to compare the performance of different hyperfeatures with single-feature as well as test our hypothesis, we trained five kinds of classifiers including (Quadratic) Discriminant, logistic regression, SVM (support vector machine), KNN (k-nearest Neighbors) and ensemble boosted tree using PSD (power spectrum density) and inter- and intra- PLV value for each trial respectively at different bands respectively.

\section{RESULTS}

\section{A. ERP}

In Fig.2, we compared conditions in changing response turns which means one of the paired participants makes the first response in the condition 1 and the other participant gives the first response in the condition 2. It can be seen that N250 ERP component enhances in the first response participant turn with the earlier expectation than the second response participant, which could be taken as the relationship between EEG and human emotion states. Moreover, although the evoked components did not vary in accordance with each participant, the time-locked components varies consistently. We could also get the early ERP components N250, N400, late ERP component P1500 and N1500. N250 is sensitive to face identity and identification-related processes [44] and $\mathrm{N} 250$ and N400 are independent (have no direct correlation) in face perception [45], [46].

\section{B. Synchronization-PLV}

We investigated the intra- and inter-synchronization across different frequency bands for two conditions, (i.e., positive and negative stimuli). The corresponding relationship between the name of channels and the number of channels is indicated in Fig.3. The Fig. 4 and Fig 5 show the intra-synchrony across different bands for two conditions while Fig.6 shows the intersynchrony across different bands for two cases.

According to the ANOVA analyses, the PLV is significantly different between different bands for the negative stimulus
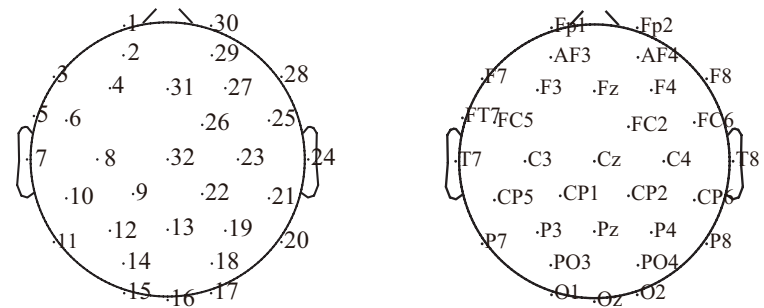

Fig. 3. The corresponding relationship between the name of channels and the number of channels.
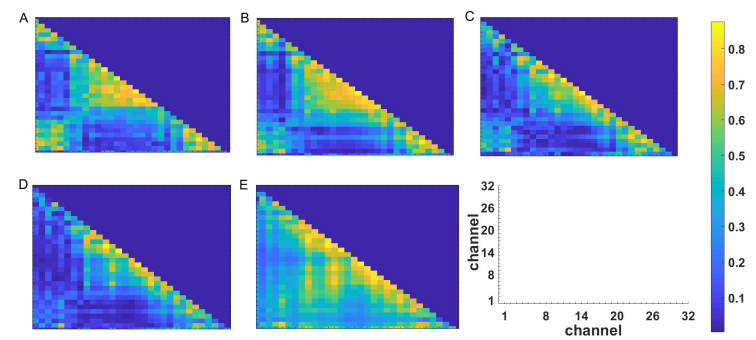

Fig. 4. Intra-synchronization - PLV matrix of participants at the negative stimuli. Sub-figures indicate delta, theta, alpha, beta and gamma frequency PLV (from A to E) respectively.

trials $\left[F(4,2475)=32.19, p<10^{-8}\right]$. As shown in Fig.4 , the bigger PLV cluster was located in the parietal area of the brain across different frequency bands. The larger intraparticipant synchronization in the beta and delta bands was found in frontal area and was found in occipital area for the theta, alpha, and gamma bands. A significant difference was observed in all five frequency bands, but the highest PLV value was found in the gamma band.

For the perception of positive emotional video stimuli, the higher PLV value cluster was in the parietal area across the five different frequency bands (shown in the Fig. 5). In low frequency bands like delta and theta, some channels in the frontal area have high synchronization with the distribution of occipital area channels. From the ANOVA result $\left[F(4,2475)=25.93, p<10^{-8}\right]$, we can see the synchronization between each pair of channels indicated a significant difference across all the five frequency bands and theta and gamma frequency bands have higher PLV values. Compared with the negative stimuli condition, the PLV values calculated by positive emotional video stimuli are similar in lower frequency bands such as delta, theta and alpha and smaller in beta and gamma bands.

According to the ANOVA statistical analysis, we found there were no significant differences in delta, theta, alpha and beta bands for positive or negative stimulus perception $(p>0.05)$ while the PLV has significant difference in gamma frequency band for the two types of stimuli $(\mathrm{p}<0.05)$.This implies that intra-PLV can be used to distinguish different conditions.

We investigated the inter-brain synchronization. As shown in Fig. 6, the gamma frequency band indicated an enhanced inter-brain synchronization. However, we did not find any 

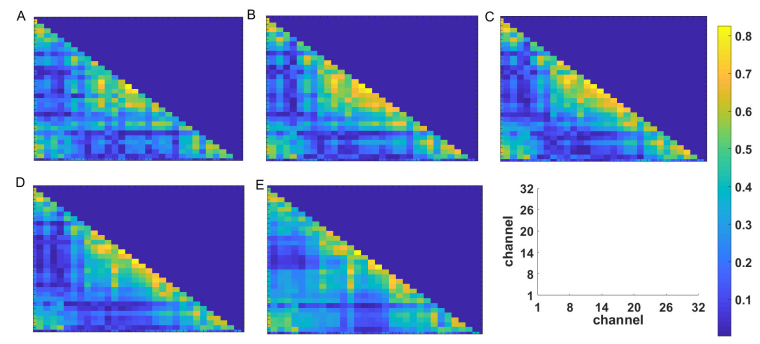

Fig. 5. Intra-synchronization PLV matrix of participants at the positive stimuli. Sub-figures indicate delta, theta, alpha, beta and gamma frequency PLV (from A to E) respectively.

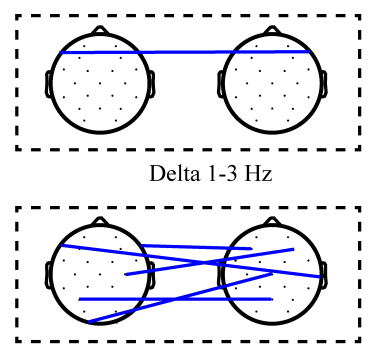

Alpha $8-12 \mathrm{~Hz}$

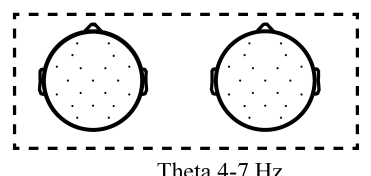

Theta $4-7 \mathrm{~Hz}$

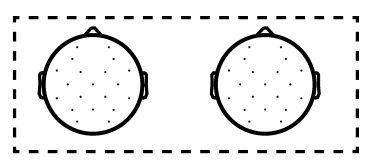

Beta $13-30 \mathrm{~Hz}$

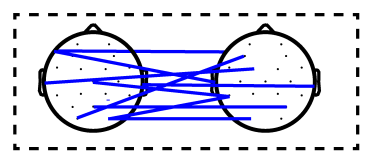

Gamma $31-40 \mathrm{~Hz}$

Fig. 6. Statistically significant inter-brain connectivity links are shown for negative vs positive in different frequency bands.

significant difference in the alpha and beta bands.

\section{Classification}

All channels were used for feature extraction. Principal component analysis (PCA) was used to reduce feature dimension after the feature extraction. The parameters in PCA were set as component reduction criterion of specifying explained variance and explained variance percentage of $95 \%$. Then five classifiers were employed to obtain classification accuracies. The KNN method has a relatively consistent performance in terms of classification accuracy when the PSD features were used (see Fig. 7). The KNN performed better in terms of classification accuracy on PLV features (Fig. 8). The KNN and ensemble boosted tree performed better in terms of classification accuracy on hyper-PLV features (Fig. 9). The classification on hyper-PLV outperformed the PLV features. The classifiers parameters are set to KNN (number of neighbors: 5, distance: euclidean distance), ensemble boosted tree (maximum number of splits:20, number of learners: 30 and learning rate: 0.1). Evaluation of classification performances was achieved by the leave-one-out cross validation.

\section{DISCUSSION AND CONCLUSION}

In this paper, we have investigated the following questions: (i) For the time-locked characteristics of EEG in emotional

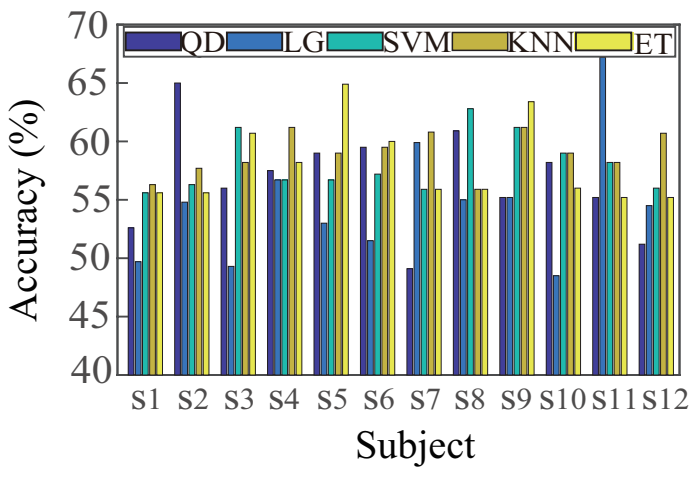

Fig. 7. Classification accuracy in QD (quadratic discriminant), LR (logistic regression), SVM, KNN and ET (ensemble boosted tree) for each participant (s is abbreviation for subject here) using PSD features at delta, theta, alpha, beta and gamma frequency bands.

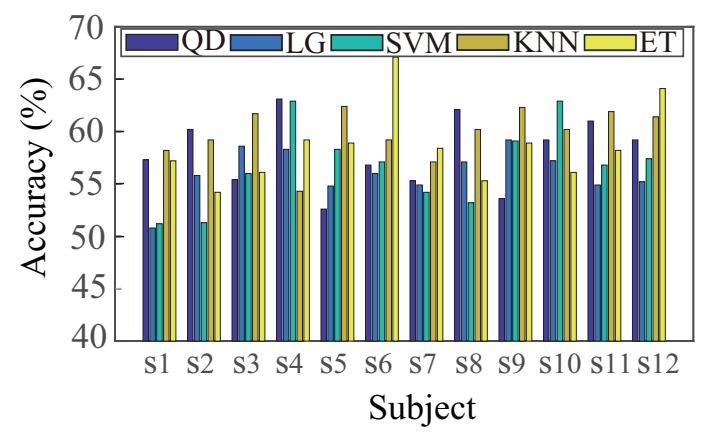

Fig. 8. Classification accuracy in QD, LR, SVM, KNN and ET for each participant using PLV features at delta, theta, alpha, beta and gamma frequency bands.

perception, are the brains of the participants influenced by the behavioral decisions? (ii) For a given emotional task, how are the brains of the paired participants intra- and intersynchronized? and (iii) What is the efficacy of features and hyper-features for emotion classification in the context of emotional interactions between subjects?

We proposed an EEG-based hyper-scanning experiment to explore the neural mechanism of emotional perception in section 2.1. As shown in Fig.1, we recorded the simultaneous EEG between a pair of subjects and put the behavioral control of the response order. We explored the ERP to investigate the effect of response order of participants. N250 amplitudes in most channels were enhanced for participants who should first give responses.

To find out the intra- and inter- synchronization mechanisms in emotional perception, we applied the PLV method to calculate the phase synchronization. The intra-connections matrices are symmetric and we got these metrics for each participant. The analysis of variance has been used to compare the PLV values between conditions for different frequency bands. We found that negative stimuli results in significantly higher synchronization as compared to positive stimuli. Lower intra-brain synchrony under positive stimuli display might be explained by that participants would be influenced more when they 


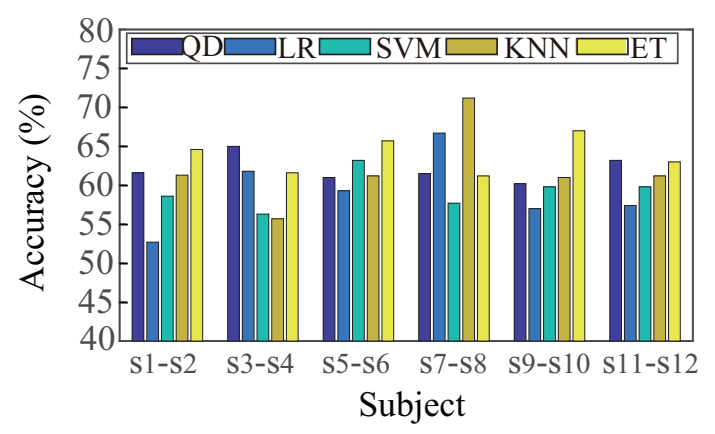

Fig. 9. Classification accuracy in QD, LR, SVM,KNN and ET for each pair participant using PLV features at delta, theta, alpha, beta and gamma frequency bands.

watched negative facial expressions. For inter-synchronization, we compared PLV values between the paired participants and found that the dominant value differences were observed in the gamma frequency band. Therefore, features derived from the gamma frequency band can be a good choice for classifying emotion perception in the context of the hyper-scanning.

In the classification analyses, we employed five classifiers, quadratic discriminant, logistic regression, SVM, KNN and ensemble boosted tree to distinguish positive and negative emotions using the PLV and PSD features and compared their classification performances. We also compared feature efficacy and found that hyper-PLV feature is better for the classification.

In this study, we have provided a basic framework towards a more comprehensive study to assess social emotional perception in the context of a multi-subject EEG hyper-scanning. In the future, we plan to collect more data and apply this technique to in a real-time social brain-computer- interface.

\section{REFERENCES}

[1] K. Löwith, "L. feuerbach und der ausgang der klassischen deutschen philosophie," 1928.

[2] G. Dumas, F. Lachat, J. Martinerie, J. Nadel, and N. George, "From social behaviour to brain synchronization: review and perspectives in hyperscanning," Irbm, vol. 32, no. 1, pp. 48-53, 2011.

[3] S. E. Barkan, Sociology: Understanding and changing the social world. Flat World Knowledge, Incorporated, 2011.

[4] J. S. House, K. R. Landis, and D. Umberson, "Social relationships and health," Science, vol. 241, no. 4865, pp. 540-545, 1988.

[5] J. Holt-Lunstad, T. B. Smith, and J. B. Layton, "Social relationships and mortality risk: a meta-analytic review," PLoS medicine, vol. 7, no. 7, p. e1000316, 2010.

[6] F. Babiloni and L. Astolfi, "Social neuroscience and hyperscanning techniques: past, present and future," Neuroscience \& Biobehavioral Reviews, vol. 44, pp. 76-93, 2014.

[7] X. Cui, D. M. Bryant, and A. L. Reiss, "Nirs-based hyperscanning reveals increased interpersonal coherence in superior frontal cortex during cooperation," Neuroimage, vol. 59, no. 3, pp. 2430-2437, 2012.

[8] B. King-Casas, D. Tomlin, C. Anen, C. F. Camerer, S. R. Quartz, and P. R. Montague, "Getting to know you: reputation and trust in a twoperson economic exchange," Science, vol. 308, no. 5718, pp. 78-83, 2005.

[9] U. Hasson, A. A. Ghazanfar, B. Galantucci, S. Garrod, and C. Keysers, "Brain-to-brain coupling: a mechanism for creating and sharing a social world," Trends in cognitive sciences, vol. 16, no. 2, pp. 114-121, 2012.

[10] K. Yun, K. Watanabe, and S. Shimojo, "Interpersonal body and neural synchronization as a marker of implicit social interaction," Scientific reports, vol. 2, p. 959, 2012.
[11] L. Bonnet, F. Lotte, and A. Lécuyer, "Two brains, one game: design and evaluation of a multiuser bci video game based on motor imagery," IEEE Transactions on Computational Intelligence and AI in games, vol. 5, no. 2, pp. 185-198, 2013.

[12] P. R. Montague, G. S. Berns, J. D. Cohen, S. M. McClure, G. Pagnoni, M. Dhamala, M. C. Wiest, I. Karpov, R. D. King, N. Apple et al., "Hyperscanning: simultaneous fmri during linked social interactions," 2002.

[13] F. Babiloni, L. Astolfi, F. Cincotti, D. Mattia, A. Tocci, A. Tarantino, M. Marciani, S. Salinari, S. Gao, A. Colosimo et al., "Cortical activity and connectivity of human brain during the prisoner's dilemma: an eeg hyperscanning study," in Engineering in Medicine and Biology Society, 2007. EMBS 2007. 29th Annual International Conference of the IEEE. IEEE, 2007, pp. 4953-4956.

[14] T. Funane, M. Kiguchi, H. Atsumori, H. Sato, K. Kubota, and H. Koizumi, "Synchronous activity of two people's prefrontal cortices during a cooperative task measured by simultaneous near-infrared spectroscopy," Journal of Biomedical Optics, vol. 16, no. 7, p. 077011, 2011.

[15] D. N. Saito, H. C. Tanabe, K. Izuma, M. J. Hayashi, Y. Morito, H. Komeda, H. Uchiyama, H. Kosaka, H. Okazawa, Y. Fujibayashi et al., "stay tuned: inter-individual neural synchronization during mutual gaze and joint attention," Frontiers in integrative neuroscience, vol. 4, p. 127, 2010.

[16] S. Anders, J. Heinzle, N. Weiskopf, T. Ethofer, and J.-D. Haynes, "Flow of affective information between communicating brains," Neuroimage, vol. 54, no. 1, pp. 439-446, 2011.

[17] G. Dumas, J. Nadel, R. Soussignan, J. Martinerie, and L. Garnero, "Interbrain synchronization during social interaction," PloS one, vol. 5, no. 8, p. e12166, 2010.

[18] F. D. V. Fallani, V. Nicosia, R. Sinatra, L. Astolfi, F. Cincotti, D. Mattia, C. Wilke, A. Doud, V. Latora, B. He et al., "Defecting or not defecting: how to read human behavior during cooperative games by eeg measurements," PloS one, vol. 5, no. 12, p. e14187, 2010.

[19] D. Chung, K. Yun, and J. Jeong, "Decoding covert motivations of free riding and cooperation from multi-feature pattern analysis of eeg signals," Social cognitive and affective neuroscience, vol. 10, no. 9, pp. 1210-1218, 2015.

[20] J. Toppi, G. Borghini, M. Petti, E. J. He, V. De Giusti, B. He, L. Astolfi, and F. Babiloni, "Investigating cooperative behavior in ecological settings: an eeg hyperscanning study," PloS one, vol. 11, no. 4, p. e0154236, 2016.

[21] W. D. Casebeer and P. S. Churchland, "The neural mechanisms of moral cognition: A multiple-aspect approach to moral judgment and decisionmaking," Biology and philosophy, vol. 18, no. 1, pp. 169-194, 2003.

[22] H. Tang, X. Mai, S. Wang, C. Zhu, F. Krueger, and C. Liu, "Interpersonal brain synchronization in the right temporo-parietal junction during faceto-face economic exchange," Social cognitive and affective neuroscience, vol. 11, no. 1, pp. 23-32, 2015.

[23] G. Emonds, C. H. Declerck, C. Boone, E. J. Vandervliet, and P. M. Parizel, "Comparing the neural basis of decision making in social dilemmas of people with different social value orientations, a fmri study." Journal of Neuroscience, Psychology, and Economics, vol. 4, no. 1, p. 11, 2011.

[24] P. YE, R. ZHU, H. TANG, X. MAI, and C. LIU, "The application of functional near-infrared spectroscopy in social cognitive neuroscience," Advances in Psychological Science, vol. 25, no. 5, pp. 731-741, 2017.

[25] M. Strait and M. Scheutz, "What we can and cannot (yet) do with functional near infrared spectroscopy," Frontiers in neuroscience, vol. 8, p. 117,2014

[26] F. Wallois, M. Mahmoudzadeh, A. Patil, and R. Grebe, "Usefulness of simultaneous eeg-nirs recording in language studies," Brain and language, vol. 121, no. 2, pp. 110-123, 2012.

[27] X. Cui, S. Bray, D. M. Bryant, G. H. Glover, and A. L. Reiss, "A quantitative comparison of nirs and fmri across multiple cognitive tasks," Neuroimage, vol. 54, no. 4, pp. 2808-2821, 2011.

[28] P. Pouliot, J. Tremblay, M. Robert, P. Vannasing, F. Lepore, M. Lassonde, M. Sawan, D. K. Nguyen, and F. Lesage, "Nonlinear hemodynamic responses in human epilepsy: a multimodal analysis with fnirseeg and fmri-eeg," Journal of neuroscience methods, vol. 204, no. 2, pp. 326-340, 2012.

[29] R. A. Ramadan and A. V. Vasilakos, "Brain computer interface: control signals review," Neurocomputing, vol. 223, pp. 26-44, 2017.

[30] S. Moscovici, Social influence and social change. Academic Press, 1976, vol. 10 . 
[31] C. Babiloni, P. Buffo, F. Vecchio, N. Marzano, C. Del Percio, D. Spada, S. Rossi, I. Bruni, P. M. Rossini, and D. Perani, "Brains in concert: frontal oscillatory alpha rhythms and empathy in professional musicians," Neuroimage, vol. 60, no. 1, pp. 105-116, 2012.

[32] C. Babiloni, F. Vecchio, F. Infarinato, P. Buffo, N. Marzano, D. Spada, S. Rossi, I. Bruni, P. M. Rossini, and D. Perani, "Simultaneous recording of electroencephalographic data in musicians playing in ensemble," cortex, vol. 47, no. 9, pp. 1082-1090, 2011.

[33] M. A. Acquadro, M. Congedo, and D. De Riddeer, "Music performance as an experimental approach to hyperscanning studies," Frontiers in human neuroscience, vol. 10, p. 242, 2016.

[34] K. Horley, L. M. Williams, C. Gonsalvez, and E. Gordon, "Social phobics do not see eye to eye:: A visual scanpath study of emotional expression processing," Journal of anxiety disorders, vol. 17, no. 1, pp. 33-44, 2003.

[35] S. Anders, F. Eippert, S. Wiens, N. Birbaumer, M. Lotze, and D. Wildgruber, "When seeing outweighs feeling: a role for prefrontal cortex in passive control of negative affect in blindsight," Brain, vol. 132, no. 11, pp. 3021-3031, 2009.

[36] S. Makeig, S. Debener, J. Onton, and A. Delorme, "Mining event-related brain dynamics," Trends in cognitive sciences, vol. 8, no. 5, pp. 204-210, 2004.

[37] S. Makeig, J. Onton et al., "Erp features and eeg dynamics: an ica perspective," Oxford Handbook of Event-Related Potential Components. New York, NY: Oxford, 2009.

[38] A. P. Burgess, "On the interpretation of synchronization in eeg hyperscanning studies: a cautionary note," Frontiers in human neuroscience, vol. 7, p. 881, 2013.

[39] M. Vinck, F. P. Battaglia, T. Womelsdorf, and C. Pennartz, "Improved measures of phase-coupling between spikes and the local field potential," Journal of computational neuroscience, vol. 33, no. 1, pp. 53-75, 2012.

[40] J.-P. Lachaux, E. Rodriguez, J. Martinerie, and F. J. Varela, "Measuring phase synchrony in brain signals," Human brain mapping, vol. 8, no. 4, pp. 194-208, 1999.

[41] H. Candra, M. Yuwono, R. Chai, A. Handojoseno, I. Elamvazuthi, H. T. Nguyen, and S. Su, "Investigation of window size in classification of eeg-emotion signal with wavelet entropy and support vector machine," in Engineering in Medicine and Biology Society (EMBC), 2015 37th Annual International Conference of the IEEE. IEEE, 2015, pp. 72507253.

[42] J.-Y. Zhu, W.-L. Zheng, Y. Peng, R.-N. Duan, and B.-L. Lu, "Eeg-based emotion recognition using discriminative graph regularized extreme learning machine," in Neural Networks (IJCNN), 2014 International Joint Conference on. IEEE, 2014, pp. 525-532.

[43] Y. Lu, W.-L. Zheng, B. Li, and B.-L. Lu, "Combining eye movements and eeg to enhance emotion recognition." in IJCAI, vol. 15, 2015, pp. $1170-1176$.

[44] S. R. Schweinberger, V. Huddy, and A. M. Burton, "N250r: a faceselective brain response to stimulus repetitions," Neuroreport, vol. 15, no. 9, pp. 1501-1505, 2004.

[45] M. F. Neumann and S. R. Schweinberger, "N250r and n400 erp correlates of immediate famous face repetition are independent of perceptual load," Brain research, vol. 1239, pp. 181-190, 2008.

[46] S. Nasr and H. Esteky, "A study of n250 event-related brain potential during face and non-face detection tasks," Journal of vision, vol. 9, no. 5, pp. 5-5, 2009. 